\title{
The influence of molecular mobility on the properties of networks of gold nanoparticles and organic ligands
}

\author{
Edwin J. Devid ${ }^{\ddagger 1}$, Paulo N. Martinho ${ }^{\ddagger 2,3}$, M. Venkata Kamalakar ${ }^{4}$, Úna Prendergast ${ }^{5}$, \\ Christian Kübel ${ }^{2,6}$, Tibebe Lemma ${ }^{5}$, Jean-François Dayen ${ }^{7}$, Tia. E. Keyes ${ }^{\star} 5$, \\ Bernard Doudin ${ }^{*}$, Mario Ruben ${ }^{* 2,7}$ and Sense Jan van der Molen ${ }^{* 1}$
}

\section{Full Research Paper}

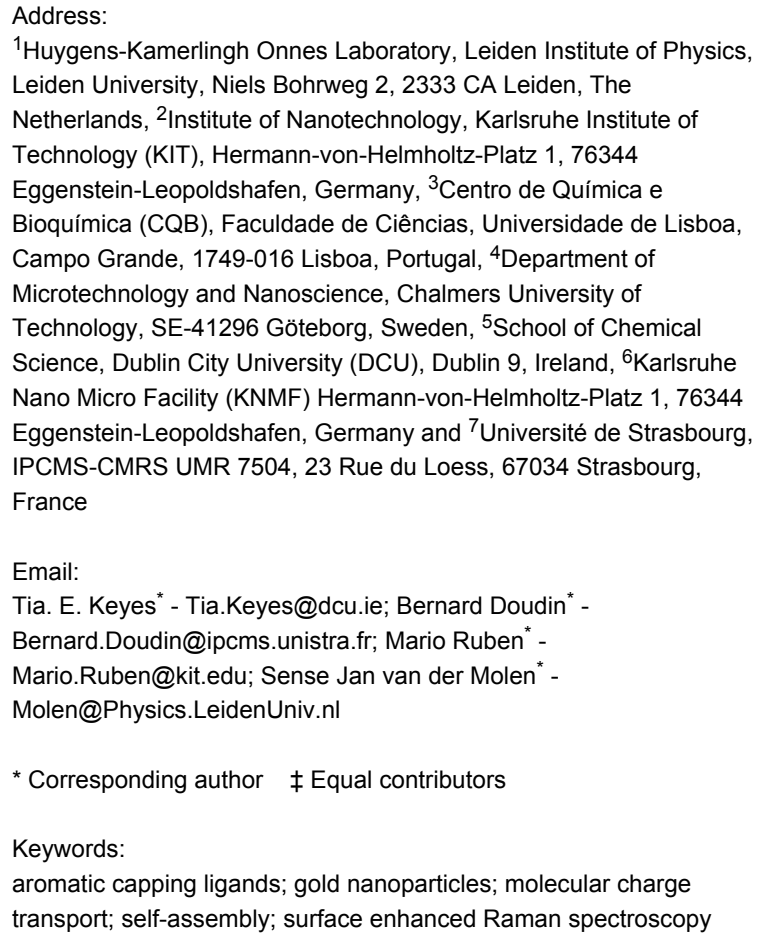

Beilstein J. Nanotechnol. 2014, 5, 1664-1674. doi:10.3762/bjnano.5.177

Received: 28 May 2014 Accepted: 26 August 2014 Published: 29 September 2014

This article is part of the Thematic Series "Molecular materials - towards quantum properties".

Associate Editor: J. Sagiv

(C) 2014 Devid et al; licensee Beilstein-Institut. License and terms: see end of document.

\begin{abstract}
We prepare and investigate two-dimensional (2D) single-layer arrays and multilayered networks of gold nanoparticles derivatized with conjugated hetero-aromatic molecules, i.e., $S$-(4-\{[2,6-bipyrazol-1-yl)pyrid-4-yl]ethynyl $\}$ phenyl)thiolate (herein S-BPP), as capping ligands. These structures are fabricated by a combination of self-assembly and microcontact printing techniques, and are characterized by electron microscopy, UV-visible spectroscopy and Raman spectroscopy. Selective binding of the S-BPP molecules to the gold nanoparticles through $\mathrm{Au}-\mathrm{S}$ bonds is found, with no evidence for the formation of $\mathrm{N}-\mathrm{Au}$ bonds between the pyridine or pyrazole groups of BPP and the gold surface. Subtle, but significant shifts with temperature of specific Raman S-BPP modes are also observed. We attribute these to dynamic changes in the orientation and/or increased mobility of the molecules on the
\end{abstract}


gold nanoparticle facets. As for their conductance, the temperature-dependence for S-BPP networks differs significantly from standard alkanethiol-capped networks, especially above $220 \mathrm{~K}$. Relating the latter two observations, we propose that dynamic changes in the molecular layers effectively lower the molecular tunnel barrier for BPP-based arrays at higher temperatures.

\section{Introduction}

Inspired by nature, self-assembly is a bottom-up method to fabricate structures at all scales from nanometer-sized ingredients. In this way, new functional materials can be created with properties that are, in principle, based on the specific functionality of their building blocks [1]. An interesting approach, used for molecular conductance experiments, includes nanoparticles (ca. $10 \mathrm{~nm}$ ) incorporated to bridge the size gap between macroscopic electrodes (larger than $100 \mathrm{~nm}$ ) and molecules (ca. $1 \mathrm{~nm}$ ) [2-8]. Typically, 2D arrays of gold nanoparticles capped by alkanethiols are created, after which dithiolated conjugated molecules are allowed to form molecular bridges between neighboring nanoparticles $[6,8]$. Although molecular insertion cannot be driven to completeness for thermodynamic reasons $[9,10]$, this protocol has proven successful in molecular electronics, e.g., by providing access to switchable molecular devices $[11,12]$. The process can also be used for non-thiol ligands [13]. Here, we extend this self-assembly procedure beyond alkanes, making use of an attractive class of molecular ligands.

Molecules of the tridentate 2,6-bi(pyrazolyl)pyridine (BPP) group are well known to act as weak $\sigma$-donor/ $\pi$-acceptor ligands exhibiting an octahedral coordination environment with a coordination number of six for transition metals [14]. Moreover, in the case of iron(II) ions, the BPP-ligands adjust the ligand field strength to access the so-called ST or spin crossover (SCO) regime [15], in which the physical properties depend strongly on their intrinsic low and high spin states (LS, S $=0$ and HS; S $=2$ ). Integrated spin transition (ST) units may be considered as potential electronic components in the construction of switching molecular devices [16,17], a vision for which the control of the attaching of BPP-units to gold nanoparticles sets the stage. Towards this goal, the synthetic introduction of substituents at the 4'-position of the pyridine moiety of BPP has been shown to be a useful strategy. In particular, the introduction of highly conductive $\pi$-conjugated phenylethynyl linker moieties with acetyl-protected thiol anchoring groups facilitates the contact to noble and coinage metal electrodes [18].

In this study, we report on the fabrication of 2D single-layer ligand-gold nanoparticle arrays (and multilayer ligand-gold nanoparticle networks) formed by gold nanoparticles covered by planar aromatic organic ligand-based molecules, namely $S$-(4-\{[2,6-bipyrazol-1-yl)pyrid-4-yl]ethynyl $\}$ phenyl)thiolate
(S-BPP). The inclusion of the thioacetate end group (see SAcBBP molecule, Scheme 1) [18] is expected to steer the adsorption of the S-BPP molecule to the gold nanoparticles [19]. The results of the structural and spectroscopic characterisation of the synthesized 2D ligand-gold nanoparticle arrays (in short Au-NP-S-BPP-arrays), by means of UV-vis and electron microscopy (SEM, HRTEM and 3D TEM) experiments, will be presented. Specifically, surface enhanced Raman spectroscopy (SERS) provides insight into the selectivity of the bond formation. Remarkably, Raman experiments also reveal subtle shifts in some S-BPP modes related to reversible structural modification within the array induced by temperature. This observation is compared to temperature-dependent transport experiments. For this purpose, the fabricated 2D Au-NP-S-BPP arrays are electrically contacted to lithographically defined devices $[5,8,9,12]$ and the obtained conductance measurements are compared to benchmark networks formed with alkanethiols spacers.

\section{Experimental \\ Capping of gold nanoparticles with S-BPP molecules}

To create arrays of S-BPP-capped gold nanoparticles, the wellestablished procedure to make alkanethiol-based nanoparticle arrays $[8,9,20]$ is adapted and applied. The first step is the synthesis of citrate-stabilized gold nanoparticles in aqueous solvent (see Supporting Information File 1, experimental section (part 1)). This is followed by centrifuging $5 \mathrm{~mL}$ of monodispersed citrate-stabilized gold nanoparticle suspension at 15000 RPM for $1 \mathrm{~h}$. After centrifugation, the aqueous solvent is removed from the settled gold nanoparticles, followed immediately by the redispersion of the nanoparticles in ethanol. Through forceful shaking, the nanoparticles are redispersed quickly to yield a stable colloidal dispersion, which is sealed in a glass bottle and sonicated in a water bath for approximately one hour.

Functionalization of gold nanoparticles with S-BPP molecules [18] is accomplished in two steps. First, $14.5 \mathrm{mg}$ of S-BPP molecules is added to a sealable glass bottle with $2 \mathrm{~mL}$ of ethanol and heated in a water bath to $70{ }^{\circ} \mathrm{C}$, under stirring until complete dissolution of the S-BPP molecules. Second, the hot S-BPP ethanolic solution is quickly added to the ethanolic dispersion of gold nanoparticles and sealed with a lid. The 
process of functionalization is accelerated by sonication in a water bath. After $15 \mathrm{~min}$, the dispersion of functionalized gold nanoparticles has changed colour. As shown below, UV-visible absorption spectroscopy reveals that this colour change can be attributed to the S-BPP molecule assembling around the gold nanoparticles. Next, the functionalized gold nanoparticles are left to settle down by gravity in a cold, dark storage environment $[21,22]$ for three days. The supernatant is then removed from the sediment, which is redispersed in $4 \mathrm{~mL}$ of chloroform and sonicated for $1 \mathrm{~h}$. Finally, a lightly purple coloured dispersion of functionalized gold nanoparticles is used to prepare a self-assembled 2D Au-NP-S-BPP array.

\section{Fabrication of 2D Au-NP-S-BPP arrays}

The 2D single-layer Au-NP-S-BPP array is prepared by using a Langmuir-Schaefer (LS) method (Scheme 1) in which a singlelayer array of functionalized gold nanoparticles is self-assembled at the air-water interface $[8,23,24]$ (see Supporting Information File 1, Figure S5). A Teflon mould, containing a hole, is filled with $300 \mu \mathrm{L}$ of demineralised Millipore water. Then, $30 \mu \mathrm{L}$ of S-BPP functionalized gold nanoparticles in chloroform are dispersed on the aqueous layer. The steadily evaporating chloroform leaves the nanoparticles at the air-water interface. The hydrophobically functionalized gold nanoparticles attract each other on the water surface and self-assemble into a highly ordered array. The array is then transferred onto a $\mathrm{Si}$ wafer substrate (covered with $300 \mathrm{~nm}$ of $\mathrm{SiO}_{2}$ ) by using a polydimethylsiloxane (PDMS) microcontact printing method [8] Other suitable types of substrates for the microcontact printing of these samples are glass, quartz, several types of plastics (like polyethylene, polypropylene foils and polyimide (Kapton) films) and electron beam lithography-written high-aspect-ratio (HAR) nanotrench electrodes devices [25]. The Au-NP-S-BPP arrays are stored in a dark and cold environment and can be kept for several months.

\section{Results and Discussion Imaging of Au-NP-S-BPP arrays and networks}

Scanning transmission electron microscopy (SEM) is used to image the arrays on flat (oxidized) silicon substrates and the Au-NP-S-BPP networks on nanotrench electrodes devices (see Supporting Information File 1, Figure S8). Scanning transmission electron microscopy (STEM) and transmission electron microscopy (HAADF-STEM, HRTEM and 3D TEM) are also used to accurately characterise the nanoscale structuring of the multilayered networks on carbon-covered TEM grids. It should be noted that, whereas regular $2 \mathrm{D}$ structures are readily obtained on flat (oxidized) silicon substrates, the ordered assembly on the TEM grids turned out to be more challenging, resulting in structures with local ordering only.

Figure 1a shows a SEM image of a nanoparticle array that was microcontact printed on a $\mathrm{SiO}_{2}$ substrate. Typically, these Au-NP-S-BPP arrays reveal ordered structures on flat surfaces extending over several hundred nanometers, depending on the microcontact printing procedure used. The nanoparticles have not coalesced as they are well-separated by the capping ligands S-BPP.

HAADF-STEM images of Au-NP-S-BPP networks (see Figure $1 \mathrm{~b}$ and Supporting Information File 1, Figure S6) show a fairly uniform distribution of functionalized gold nanoparticles, sometimes as a single layer, but typically as a few stacked layers. The latter is probably caused by repeating deposition of Au-NP-S-BPP arrays on the TEM grids by dipping the TEM grids. Microscopic analyses of the network confirm the monodispersity of the gold nanoparticles with an average diameter of $8.5 \pm 1.5 \mathrm{~nm}$. Moreover, fast Fourier transforms (FFT) of the HAADF-STEM images indicate an average particle distance of $10.6 \mathrm{~nm}$. The resulting average next neighbour dis-

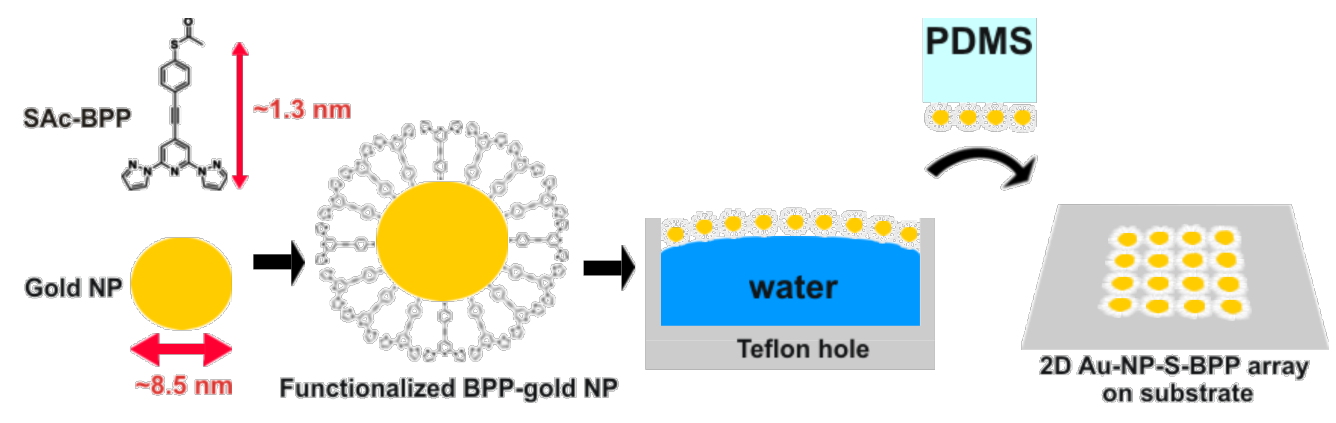

Scheme 1: Fabrication of 2D Au-NP-S-BPP array (not to scale). The ingredients are gold nanoparticles (diameter $8.5 \pm 1.5 \mathrm{~nm}$ ) and SAc-BPP molecules (length of the molecule $1.3 \mathrm{~nm}$, without the acetyl (Ac) group). The arrays are fabricated through self-assembly and then placed onto a substrate of choice by PDMS microcontact printing. 


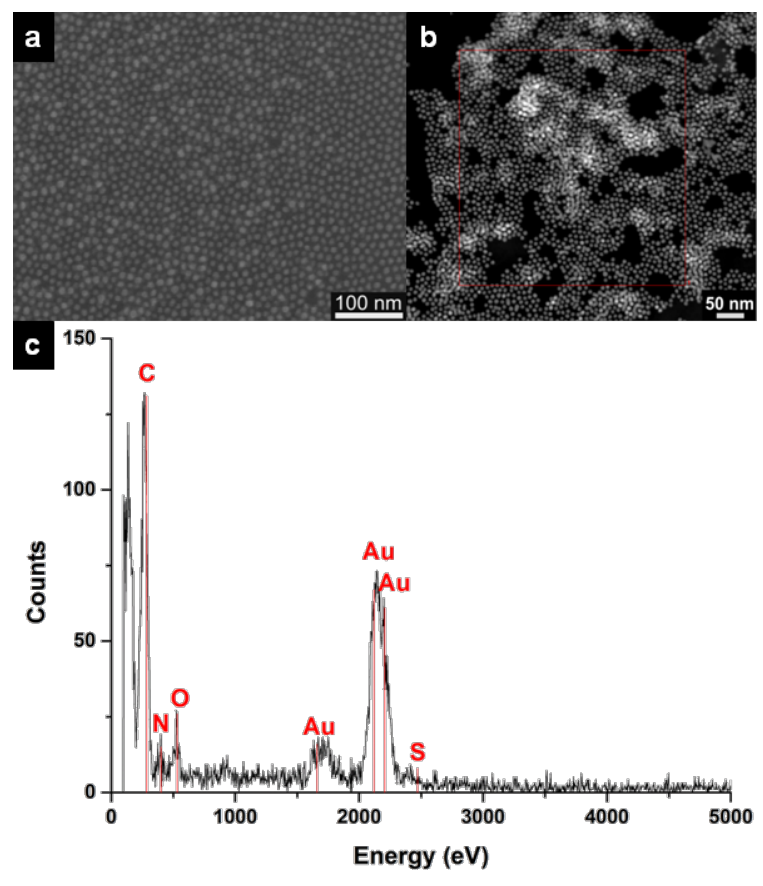

Figure 1: Characterization of Au-NP-S-BPP arrays and networks by electron microscopy. a) SEM image of a $2 \mathrm{D}$ single-layer microcontact printed Au-NP-S-BPP array on a flat $\mathrm{Si}-\mathrm{SiO}_{2}$ substrate; b) STEMreference image of a Au-NP-S-BPP network area on a TEM grid substrate; c) local EDX analysis revealing the elemental composition.

tance is close to $2 \mathrm{~nm}$, consistent with an expected value between one $(1.3 \mathrm{~nm})$ and two $(2.6 \mathrm{~nm})$ molecular lengths of S-BPP.

Figure 1c shows the energy dispersive X-ray (EDX) spectrum corresponding to the HAADF-STEM image of the thin Au-NP-S-BPP network film (see Figure 1b). In addition to Au, the expected spectra of the organic ligand elements $\mathrm{C}, \mathrm{N}$ and $\mathrm{S}$ (partially overlapped by $\mathrm{Au}$ ) are detected. The observed oxygen peak may originate from the presence of water when dipping the TEM grid into the self-assembled Au-NP-S-BPP arrays that floats on water. HAADF-STEM tomography $[26,27]$ is used to further analyse the spatial packing of multilayered Au-NP-SBPP networks (see Supporting Information File 1, Figure S7) From a 3D reconstruction of a multilayered Au-NP-S-BPP network, we find indeed that the S-BPP functionalized gold nanoparticles not only form a packed array separated by organic $\mathrm{S}-\mathrm{BPP}$ molecules in $2 \mathrm{D}$, but also enable sterically driven ordering of gold nanoparticles layers in a partial multi-stack volume.

\section{UV-vis spectroscopy}

Ultraviolet-visible (UV-vis) spectroscopy is performed to gain insight into the optical properties of these molecule-gold nanoparticle arrays, and specifically to investigate the influence of the S-BPP molecules on the effective dielectric constant. Metal nanoparticles exhibit absorbance due to surface plasmon resonances (SPR) that occur at frequencies $\omega$ (or wavelengths $\lambda$ ) at which the surface charges of the particles are oscillating resonantly with the electromagnetic driving field (the incoming photons). For nearly spherical particles, only one SPR mode is expected [28]. According to the Mie theory in the dipolar quasistatic approximation (in which the diameter $d$ of the nanoparticle is assumed to be much smaller than the wavelength, i.e., $d<<\lambda$ ), the position of the SPR is directly related to the permittivity of the medium surrounding the nanoparticle [29,30]. In molecule-gold nanoparticle arrays, this permittivity will be largely influenced by the molecules separating the nanoparticles. Hence, Au-NP-S-BPP arrays are expected to have optical properties different from reference alkanethiol-gold nanoparticle arrays. A further influence on the SPR is exerted by the optical interaction of one nanoparticle with the full set of neighbouring gold nanoparticles.

A suitable way to describe the SPR in molecule-gold nanoparticle arrays is through the Maxwell-Garnett theory (again in the quasi-static approximation). This effective medium theory defines an effective dielectric constant of the medium $\varepsilon_{\text {eff }}$ that takes into account both the presence of the surrounding medium and the neighbouring nanoparticles [31-33]. The resonance condition is then given by:

$$
\varepsilon_{1}\left(\omega_{\mathrm{sp}}\right)(1-\mathrm{f})+\varepsilon_{\mathrm{m}}(2+\mathrm{f})=0
$$

Here, $\omega_{\text {sp }}$ denotes the frequency of the SPR and $\varepsilon_{\mathrm{m}}$ is the dielectric constant of the medium surrounding the nanoparticles. The so-called filling factor $\mathrm{f}=V_{\text {clusters }} / V_{\text {total }}$ denotes the relative volume occupied by other nanoparticles around the resonating nanoparticle. In this way, the surrounding nanoparticles in an array are incorporated into the theory effectively. Note that for $\mathrm{f}=0$ (i.e., there is no interaction between the nanoparticles) the condition is $\varepsilon_{1}(\omega)=-2 \varepsilon_{\mathrm{m}}$, as in standard Mie theory.

Figure 2 shows the absorption curves for four types of 2D molecule-gold nanoparticle arrays, with alkanethiol-protected gold nanoparticles of various lengths $(\mathrm{C} 8, \mathrm{C} 10, \mathrm{C} 12)$ compared to the 2D Au-NP-S-BPP array. From C8 to C12, the SPR shifts to shorter wavelengths [9], as expected for a blue shift originating from a change in the spacing distance between the gold nanoparticles, i.e., a decrease in the filling factor $\mathrm{f}$, (see Equation 1). Note that the change in $\varepsilon_{\mathrm{m}}$ is expected to be negligible for the alkanethiol series [30,34]. However, the situation is different for the 2D Au-NP-S-BPP array, where we do expect a significant change in $\varepsilon_{\mathrm{m}}$, since S-BPP molecules are fully conjugated and hence more easily polarizable. Compared to the 
alkanethiol-gold nanoparticle arrays, a red shift is indeed observed in Figure 2. This is also confirmed experimentally from UV-vis spectroscopy of a C8-gold nanoparticle dispersion and a S-BPP-gold nanoparticle dispersion (both in chloroform), in which effectively $f=0$. In Figure S9 (see Supporting Information File 1) we show that the SPR peak of the S-BPPcovered gold nanoparticles in solution is red-shifted by $17 \mathrm{~nm}$ compared to the $\mathrm{C} 8$-gold nanoparticle dispersion. By using Mie theory $(f=0)$, we estimate the relative dielectric constants for C8-gold nanoparticle dispersion and S-BPP-gold dispersion to be $2.2 \pm 0.1$ and $2.8 \pm 0.1$, respectively.

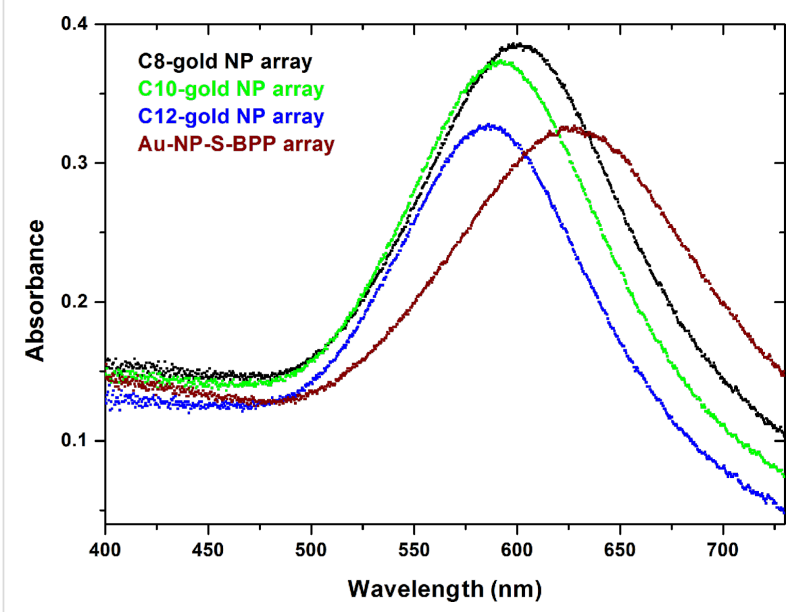

Figure 2: Surface plasmon resonance spectroscopy of several functionalized gold nanoparticle arrays studied in our work. Gold nanoparticles are covered by C8 (black), C10 (green), C12 (blue) and S-BBP (red), respectively.

The results obtained so far can now be checked for consistency. Since we are able to estimate f from the electron microscopy images, for both the $\mathrm{C} 8$-gold nanoparticle array and the Au-NP-S-BPP array, we can apply the Maxwell-Garnett theory to estimate the dielectric constant $\varepsilon_{\mathrm{m}}$ in these arrays as well. Indeed, we find approximately the same values for $\varepsilon_{\mathrm{m}}$ within an array or in dispersion, for both S-BPP molecules and for C8

Table 1: Relative dielectric constant due to the ligands $\varepsilon_{m}$ (octanethiols vs BPP ligands) as calculated from UV-vis spectra of both nanoparticle solutions and nanoparticle arrays.

\begin{tabular}{lll}
$\begin{array}{l}\text { type of functionalized gold } \\
\text { nanoparticles }\end{array}$ & $f \quad \begin{array}{l}\text { relative } \\
\text { dielectric } \\
\text { constant } \varepsilon_{m}\end{array}$ \\
\hline C8-gold nanoparticle dispersion & 0 & $2.2 \pm 0.1$ \\
S-BPP-gold nanoparticle dispersion & 0 & $2.8 \pm 0.1$ \\
C8-gold nanoparticle array & 0.35 & $2.4 \pm 0.1$ \\
Au-NP-S-BPP array & 0.36 & $2.8 \pm 0.1$
\end{tabular}

molecules, summarized in Table 1 . These values are consistent with the values for alkanethiol-gold nanoparticle arrays and oligo phenylene ethynylene (OPE)-bridged gold nanoparticle arrays obtained through molecular exchange [9,30]. We note that the latter type of arrays does contain a mixture of OPE molecules and alkanes, unlike our Au-NP-S-BPP arrays.

\section{Room-temperature Raman spectroscopy of 2D Au-NP-S-BPP arrays}

In order to get more insight into the binding of the S-BPP molecules to the gold as well as to investigate the temperaturedependence of the molecular ordering, surface enhanced Raman spectroscopy of the 2D Au-NP-S-BPP array was performed. In Figure 3 the room temperature Raman spectra of a bulk (powder) sample of S-BPP molecules (see Figure 3a) and of a 2D Au-NP-S-BPP array (see Figure 3b) are compared. In spite of the much lower concentration of the S-BPP molecule anticipated from the 2D single layer coated array (at least three orders of magnitude) compared to the bulk value, the Raman spectral intensity is comparable between the two types of samples (see

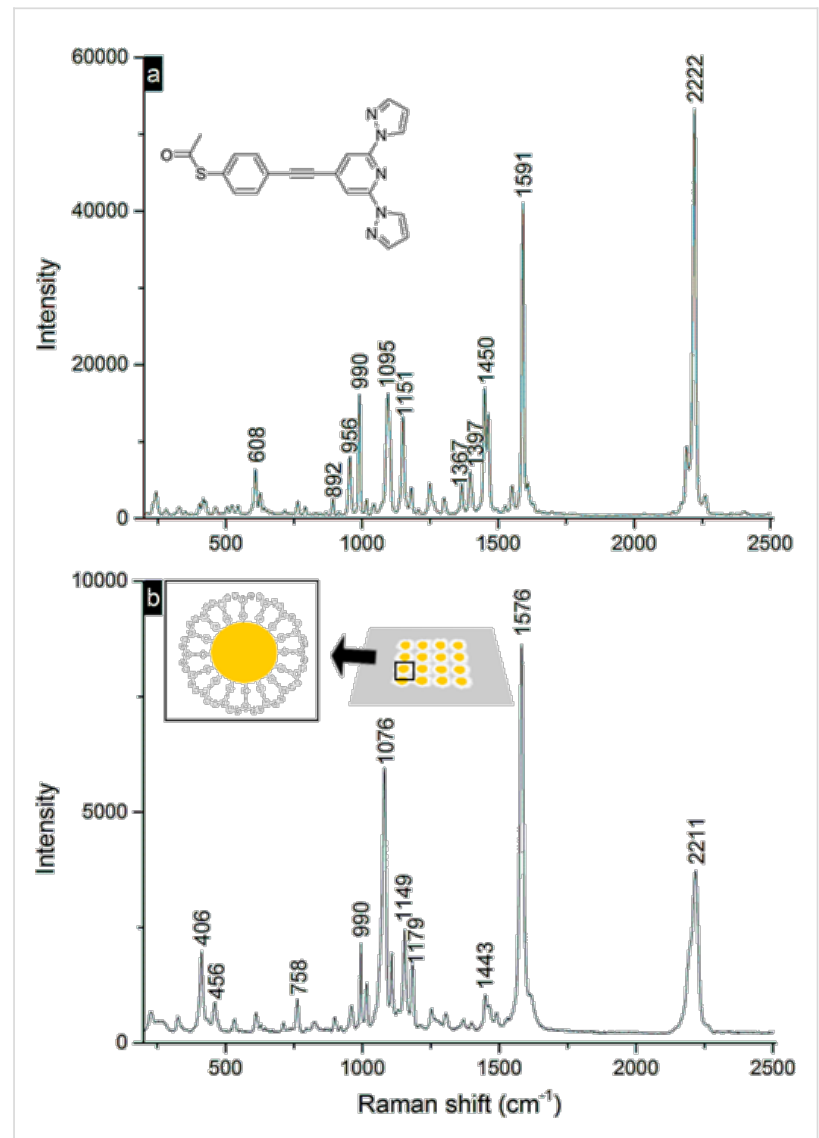

Figure 3: a) Room temperature Raman spectrum of bulk (powder) SAc-BPP molecules showing the region of $200-2400 \mathrm{~cm}^{-1}$ excited at $633 \mathrm{~nm}$ (1.2 mW illuminating power on the sample); b) Room temperature Raman spectrum of 2D (single layer) Au-NP-S-BPP array obtained by microcontact printing on a quartz substrate. 
Figure 3a vs Figure 3b). Moreover, the Raman spectra from the Au-NP-S-BPP array show a better signal-to-noise ratio attributed to surface enhancement of the Raman signal. The arrayed nature of the gold nanoparticles and their small interparticle separation leads to a plasmon absorbance shown in Figure 2, which is resonant with the $633 \mathrm{~nm}$ excitation used here. Although there are clear commonalities, the spectra of the 2D single layer array and SAc-BPP powder spectra exhibit notable differences. The individual spectral features are broader in the SERS spectrum, consistent with the higher heterogeneity in the microenvironment the S-BPP molecules experienced in the array compared to the powder. Furthermore, the SERS spectrum is less complex than that of the powder. This is consistent with plasmonic enhancement as the vibrational modes involving the atoms closest to the gold nanoparticle will be selectively enhanced.

Whereas the low frequency $\mathrm{Au}-\mathrm{S}$ or $\mathrm{Au}-\mathrm{N}$ stretch modes cannot be easily discerned from the background in the spectral region below $400 \mathrm{~cm}^{-1}$, it is interesting to note that the thioacetate and acetate modes observed in the solid sample, for example a weak feature at $1698 \mathrm{~cm}^{-1}$ assigned to the acetate $\mathrm{C}=\mathrm{O}$ and features between $1367-1380 \mathrm{~cm}^{-1}$, are no longer evident in the SERS spectrum of the Au-NP-S-BPP arrays. This strongly suggests that the thiol is bound to the gold after surface-mediated hydrolysis of the acetate group. The dominance of key benzenethiol modes in the SERS spectrum is also indicative of binding via thiol moiety. The most intense Raman feature in the powder spectrum of S-BPP is the aryl in-plane $\mathrm{C}-\mathrm{C}$ stretch mode centred at $1591 \mathrm{~cm}^{-1}$. This mode is shifted to $1576 \mathrm{~cm}^{-1}$ in the bound S-BPP molecule of the 2D arrays, which matches precisely the in-plane $\mathrm{C}-\mathrm{C}$ stretch reported for SERS of benzenethiol on copper or silver and is a further indication of binding through sulfur or thioacetate $[35,36]$.

The second most intense feature in the SERS spectrum is a mode at $1076 \mathrm{~cm}^{-1}$, which is assigned to the aromatic $\mathrm{C}-\mathrm{S}$ stretch shifted from $1095 \mathrm{~cm}^{-1}$ in the powder sample. Both the shift and enhancement of this mode is characteristic of SAMs of benzenethiol on plasmonic metals, and is further evidence that the S-BPP ligand is binding to the gold surface through this moiety [35,36]. Other characteristic benzenethiol features are also enhanced, at 990, 660 and $406 \mathrm{~cm}^{-1}$. The alkyne $\mathrm{C} \equiv \mathrm{C}$ stretch mode, which is by far the most intense mode in the powder sample, is reduced in relative intensity in the 2D singlelayer array, but remains a dominant feature albeit shifted from 2222 to $2211 \mathrm{~cm}^{-1}$ on surface binding. This suggests a modest weakening of the $\mathrm{C} \equiv \mathrm{C}$ bond presumably induced by binding of the thiol to the surface [37]. By comparison, the Raman modes from the S-BPP moiety are weaker than the benzenethiol moiety in the SAM spectrum. The features at 1443, 1149, 1179 and $785 \mathrm{~cm}^{-1}$ are attributed to the S-BPP ligand, principally to the pyrazole moieties [38]. A weak shoulder centred at $1607 \mathrm{~cm}^{-1}$ is attributed to the pyridine moiety. The low relative intensity of this mode suggests it is not directly bound to the nanoparticle. Overall, the predominance of SERS signal from the benzenethiol moiety and the comparatively weaker enhancement of pyrazole modes provide strong evidence that the S-BPP ligand binds to the nanoparticles preferably through the thiol linker.

\section{Temperature-dependent Raman spec- troscopy on 2D Au-NP-S-BPP arrays}

The studies by Raman microscopy over the temperature range 80 to $353 \mathrm{~K}$ are shown in Figure 4. The absence of significant chemical changes with temperature is indicated by the overall similarity between the spectra under temperature variation. However, some subtle changes can be noticed. Across most of the SERS-enhanced modes, a small shift to the blue of between 2 and $4 \mathrm{~cm}^{-1}$ is observed with decreasing temperature. Interestingly, the unaffected modes are the weakest features in the spectrum, i.e., those that are not strongly SERS enhanced. In other words the bonds nearest to the nanoparticle surface are most affected by the changing temperature. This observation suggests that temperature induces changes to bonding interactions between the nanoparticle and BPP. Possible changes are conformational/orientational changes of BPP with respect to the nanoparticle surface, which might be expected to cause shifts in the frequencies of bonds close to the nanoparticle. Such electronic changes would also be likely to transmit to chemical moieties conjugated to the bound group [39]. Correspondingly, the most strongly affected modes are the $\mathrm{C}-\mathrm{C}$ stretch associated with the benzenethiol at $1576 \mathrm{~cm}^{-1}$ at room temperature, which shifts to $1582 \mathrm{~cm}^{-1}$ at $80 \mathrm{~K}$ and to $1575 \mathrm{~cm}^{-1}$ at $353 \mathrm{~K}$. The alkyne $\mathrm{C} \equiv \mathrm{C}$ stretch mode is also particularly strongly affected and shifts from a value of $2207 \mathrm{~cm}^{-1}$ at $353 \mathrm{~K}$ to 2211 $\mathrm{cm}^{-1}$ at room temperature, and to $2223 \mathrm{~cm}^{-1}$ at $80 \mathrm{~K}$. This mode is broad at $353 \mathrm{~K}$, comprising a main feature and a shoulder at room temperature, which sharpens and resolves at $80 \mathrm{~K}$ into a second band at $2190 \mathrm{~cm}^{-1}$. As described previously, a significant shift in the $\mathrm{C} \equiv \mathrm{C}$ stretch mode was observed on thiol binding to the gold surface indicating the significant electronic coupling between the benzenethiol and the surface. It is noteworthy that the $\mathrm{C} \equiv \mathrm{C}$ stretch mode is so sensitive to temperature in this system, as also found in other reports on Raman of alkynes [37]. Their large polarizability renders them sensitive to electronic changes within their molecular vicinity, particularly in conjugated systems $[40,41]$. The surface-bound benzenethiol is sensitive to temperature, but the alkyne exhibits much larger temperature-dependent spectral changes, with a shift of $16 \mathrm{~cm}^{-1}$ between 80 and $353 \mathrm{~K}$. We therefore tentatively attribute this to changes in the orientation of the mole- 


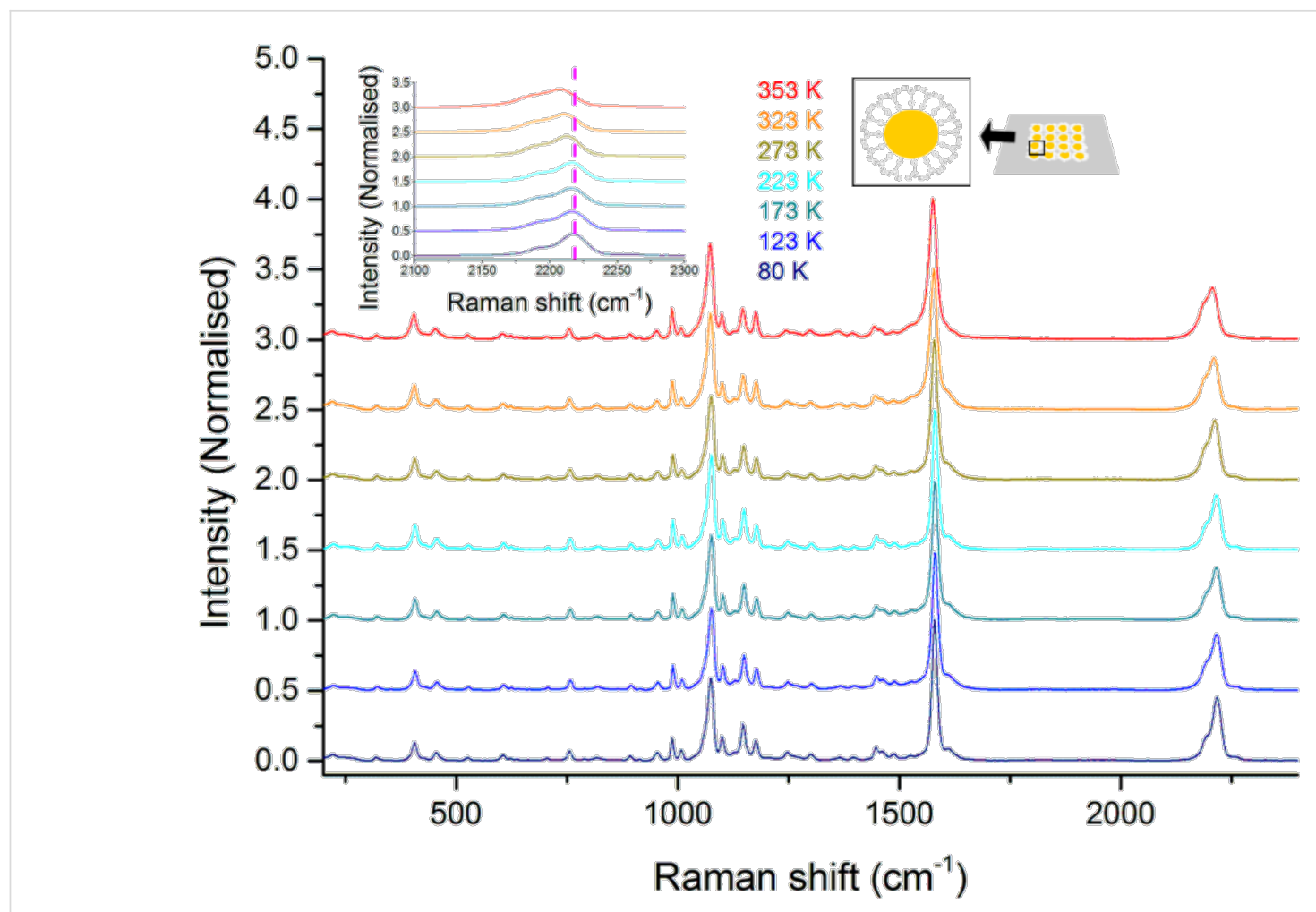

Figure 4: Temperature-dependent Raman spectra of 2D Au-NP-S-BPP arrays microcontact printed on a quartz substrate excited at $633 \mathrm{~nm}$ (illuminating power $1.2 \mathrm{~mW}$ at the sample). Inset: expansion of the alkyne stretch mode; the vertical marker emphasizes the temperature shifts in the spectra.

cule, possibly promoted by $\mathrm{Au}-\mathrm{S}$ mobility at the surface with temperature. It is important to note that the Raman spectral changes with temperature are fully reversible, i.e., restoring the $\mathrm{Au}-\mathrm{NP}-\mathrm{S}-\mathrm{BPP}$ arrays to room temperature after a cooling to 80 $\mathrm{K}$ leads to a recovery of the original room spectrum with all features.

Comparison studies on alkanethiol-modified gold nanoparticle arrays reveal that temperature changes in their associated Raman spectra are much more modest than those observed for Au-NP-S-BPP arrays (see Supporting Information File 1, Figure S10). The largest shifts observed in individual modes typically did not exceed $2 \mathrm{~cm}^{-1}$ and sharpening (improved resolution) of the vibrational bands, was the main effect of low temperature. This observation appears to validate our hypothesis that molecular structural/orientational changes related to the S-BPP ligand bound to the nanoparticle are driving the changes observed in the Raman spectrum with temperature. We note that both the magnitude and the reversibility of the Raman changes observed with temperature effectively preclude the possibility that the actual binding mode to the nanoparticle is changing. The extensive lateral interactions characteristics of alkanethiol-nanoparticle arrays make them more tightly packed than S-BPP. Therefore S-BPP is more prone to random orienta- tion and has more freedom to reorient at the nanoparticle surface promoted by temperature. The observed spatial flexibility of the thiol-anchored BPP ligands is of interest for molecule chelation purposes, as it should facilitate the envisioned complexation of $\mathrm{Fe}(\mathrm{II})$ metal ions.

\section{Conductance measurements on a multilay- ered Au-NP-S-BPP network}

Charge transport in Au-NP-S-BPP network devices provides additional experimental insight into the question of thermal stability of the nanoparticles architecture. Here, not only the S-BPP molecules, but also the nanoparticles are expected to play a role. At low temperatures, the thermal energy $k_{\mathrm{B}} T$ becomes comparable to their charging energy $E_{\mathrm{C}}=e^{2} / 2 C$, i.e., the electrostatic energy needed to add an electron onto the metallic nanoparticle (here $C$ is the total capacitance of a nanoparticle in the array and $e$ is the electron charge). In that case, Coulomb blockade will hamper charge transport. Earlier work on alkanethiol-gold nanoparticle networks demonstrated a wide range of charge transport behaviours within the Coulombblockade regime $[2,5,9,42]$. We have recently studied the crossover between the sequential tunneling and cotunneling regimes for alkanethiol networks, as well as for alkanethiol networks with dithiolated OPE-3 bridges [5]. We estimated typical 
Coulomb-blockade charging energies of around 14-17 meV [5], in correspondence with temperature- and voltage-dependent transport measurements. Hence, in alkanethiol and OPE-based networks Coulomb blockade dominates below 200-250 K, whereas around room temperature, the current-voltage $(I-V)$ characteristics are linear and practically independent of temperature.

The same method as in [5] is used to investigate the charge transport through Au-NP-S-BPP networks. We fabricate nanotrench devices with a high width-to-length aspect ratio (ca. 200) by electron beam lithography and metal lift-off. Through patterning $\mathrm{Ti}(3 \mathrm{~nm}) / \mathrm{Au}(47 \mathrm{~nm})$-electrodes of $20 \mu \mathrm{m}$ width, separated by a gap of around $100 \mathrm{~nm}$ (about 10 nanoparticles), are created on $\mathrm{Si} / \mathrm{SiO}_{2}$ substrates. We transfer the nanoparticle array onto these electrodes via a PDMS stamp. Samples containing 2D single-layer Au-NP-S-BPP arrays typically exhibited very high resistance values (more than $100 \mathrm{G} \Omega$ ). For that reason, we decided to stamp multilayers of Au-NP-SBPP networks (three times stamped). Note that the data shown below are obtained after full stabilization of the sample, following an initial resistance decrease as a function of time at room temperature. Most likely, the latter is the result of a slow re-ordering process [43-45].

Figure 5a shows the low-bias resistance vs temperature for a multilayered Au-NP-S-BPP network microcontact printed on a nanotrench device. The interparticle voltage bias, typically smaller than the device voltage bias by a factor of 10 , is of the order of $3 \mathrm{mV}$. We find that the resistance decreases monotonically with temperature over the entire temperature range, without a clear saturation at higher temperatures (see the inset of Figure 5a and Supporting Information File 1, Figure S11 for temperature dependent current-voltage $(I-V)$ curves of Au-NP-S-BPP networks).

If we compare octanethiol and OPE-based networks with the Au-NP-S-BPP network, the latter behaves differently at higher temperatures. For example, for the first two types of samples, the low-bias resistance is basically independent of voltage and temperature at room temperature, indicating that $k_{\mathrm{B}} T_{\mathrm{R}}>E_{\mathrm{C}}$ (see Supporting Information File 1, Figure S12) [5]. However, the $I-V$ curves of the Au-NP-S-BPP network are surprisingly nonlinear, with large currents occurring at larger bias. We focus our discussion on the low-bias behaviour, in the linear approximation. The particular temperature-dependent behaviour of the Au-NP-S-BPP networks becomes most clear in Figure 5b, which shows the data of Figure 5a in an Arrhenius plot. For comparison, we also display Arrhenius plots for C8 and OPE networks (taken from Supporting Information File 1, Figure S12), rather well-described by activated behaviour between $70 \mathrm{~K}$ and $300 \mathrm{~K}$ as indicated by the straight-line approximation in Figure 5. This is indeed expected for the regime of sequential tunnelling (note that for the lower-temperature cotunneling regime, Efros-Shklovskii behaviour is expected, resulting in an exponential dependence on $T^{-0.5}[2,42]$ ). For the three types of samples, there is now a clear similarity in the low-temperatures range, illustrated by the parallel lines in the semilog plot of Figure 5b, indicating an activation energy of the same order for the three molecular spacers. Nevertheless, the Au-NP-S-BPP network sample exhibits a very different behaviour for higher temperatures, showing a clear upturn in the Arrhenius plot (i.e., at lower 1/T values in Figure 5b).

A first explanation for a deviation might be that the charging energy $E_{\mathrm{C}}=e^{2} / 2 C \propto C^{-1}$ is actually higher for the Au-NP-S-
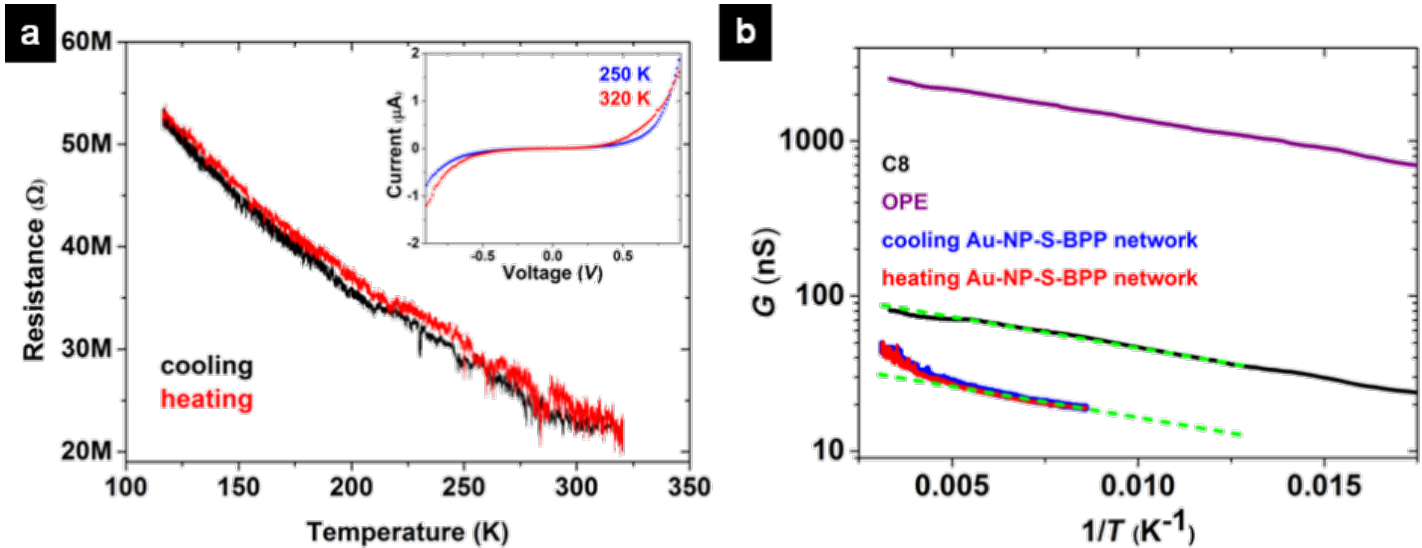

Figure 5: a) Low-bias resistance of a multilayered Au-NP-S-BPP network as a function of the temperature for $116 \mathrm{~K}<T<320 \mathrm{~K}$. Both cooling (black) and heating (red) traces are shown. Inset shows I-V curves of multilayered Au-NP-S-BPP network at $250 \mathrm{~K}$ and $320 \mathrm{~K}$; b) Arrhenius plot (semilog plot of $G$ vs $1 / T$ ) of the data in Figure 5a compared to experiments on similar, i.e., three times stamped, C8 networks (black) and OPE-bridged networks (purple). The green dashed lines are parallel guides to the eye to compare the slope of various networks. 
BPP networks than for the alkanethiols networks. This would be the case if the nanoparticle radius $R$ and/or the effective dielectric constant $\varepsilon_{\text {eff }}$ around the nanoparticles were considerably lower for the Au-NP-S-BPP networks than for the octanethiol networks (cf. the self-capacitance of a sphere: $C=4 \pi \varepsilon_{0} \varepsilon_{\mathrm{r}} R$, see also Supporting Information File 1, Figure S13 for models including nearest neighbours). However, this is not likely. First, the nanoparticles are made in the same manner for all sets of devices (independent of the molecular species investigated) and they are thus observed to be of similar size. Second, UV-vis spectroscopy indicates that $\varepsilon_{\mathrm{m}}$ (and also $\varepsilon_{\mathrm{eff}}$ which incorporates f) is actually higher for Au-NP-S-BPP networks than for alkanethiol networks (see Table 1). This should thus lead to a lower charging energy for S-BPP than for octanethiol networks and hence to linear $I-V$-curves at room temperature. Additionally, it is difficult to reconcile a Coulomb-blockade picture with the continuous increase of the slope of the Arrhenius plot of Figure 5b when heating Au-NP-S-BPP networks. The latter would suggest a significant change of the activation energy, i.e., of the charging energy, as temperature increases. For these reasons, we exclude Coulomb blockade as the reason for the upturn in Figure 5 b.

Following the results from temperature-dependent Raman spectroscopy, we propose that the deviation from simple Arrheniuslaw behaviour originates from fluctuations in the molecular interconnects, resulting in a modification of the effective tunnel barrier. Indeed, the nanoparticles are separated by loosely interacting S-BPP molecules, which cannot form a close-packed structure and hence keep room for thermally-driven motions. At higher temperatures, variations in the relative orientation of S-BPP neighbours can result in fluctuations in $\pi-\pi$ interactions, yielding changes in charge transfer probability between nanoparticles. We expect that in this situation, the effective transmission of the tunnel barrier becomes the time-average of the set of all possible configurations, each with their own specific transmission value, in a way somewhat similar to the recent proposal in [46]. For example, temporarily enhanced $\pi-\pi$ interaction should lead to higher tunnelling probabilities. Timeaveraging of such fluctuations may thus result in an enhanced transport at high temperatures, explaining the upturn in the Arrhenius plot for the S-BPP network in Figure 5b. One should point out that the transport measurements per se do not present a conclusive evidence of disorder-enhanced conductivity of nanoparticles networks. However, the combination of Raman spectroscopy and transport measurements favours such a model, proposing a new approach for understanding how disorder can impact the transport properties in molecular junctions. Additional calculations will be required to extend our hypothesis of fluctuations-enhanced transport between particles, taking into account the percolation character of transport in molecularly interconnected arrays. Complementary future experiments may use conducting-probe AFM or eutectic GaIn methods. In that case, monolayers of S-BPP formed at both electrodes could be gently brought into mechanical contact, after which temperature-dependent I(V) measurements can be done.

\section{Conclusion}

Self-assembled gold nanoparticle arrays, stabilized by a new type of conjugated organic molecules as capping ligands, can be assembled into two-dimensional arrays that form locally wellordered structures on different types of substrates. Raman spectroscopy reveals that these S-BPP molecules coordinate selectively to the nanoparticles through thiol-gold bonds, leaving the pyridine and pyrazole available for a further chemical binding. Temperature-dependent Raman measurements exhibit frequency shifts for several key modes of the S-BPP molecules, which points to changes of the molecular orientation occurring at high temperatures. The extent of structural dynamics is far greater than observed for simple alkanethiolmodified nanoparticle arrays, and is consistent with the less dense packing anticipated for the S-BPP ligands. This provides also an explanation for the peculiar temperature dependence of the electrical properties of the S-BPP networks, which shows a clear deviation from Arrhenius behaviour above $220 \mathrm{~K}$. Hence, our work suggests that the conductance behaviour of molecule-nanoparticle arrays can be tuned as an indicator of dynamical disorder in these structures, which can be a prerequisite to create nanoparticle-network candidates for further chemical functionalization or reactivity. This sets the stage for the attractive possibility of a coordination with $\mathrm{Fe}(\mathrm{II})$ metal ions, thereby introducing switchable spin transition units into the network.

\section{Supporting Information}

\section{Supporting Information File 1}

Additional experimental data.

[http://www.beilstein-journals.org/bjnano/content/ supplementary/2190-4286-5-177-S1.pdf]

\section{Acknowledgements}

Velimir Meded, Rodrigo Prieto-Gonzales and Jan van Ruitenbeek are gratefully acknowledged for fruitful discussions and giving valuable advice. This work is partly supported by the NanoSci-E+ ERA program INTERNET and by the Netherlands Organization for Scientific Research via a NWO-Vidi grant (SJvdM). Technical support from the STnano facilities and Labex NIE 11-LABX-0058_NIE are also gratefully acknowledged. T. E. K., Ú. P. and T. L. gratefully acknowledge the Irish Government's Programme for Research in Third-Level Institu- 
tions, Cycle 4, Ireland's EU Structural Funds Programmes 2007-2013 and a Career Enhancement and Mobility Fellowship cofunded by Marie Curie Actions.

\section{References}

1. Whitesides, G. M.; Grzybowsky, B. Science 2002, 295, 2418-2421. doi:10.1126/science.1070821

2. Tran, T. B.; Beloborodov, I. S.; Lin, X. M.; Bigioni, T. P.; Vinokur, V. M.; Jaeger, H. M. Phys. Rev. Lett. 2005, 95, 0768061-0768064. doi:10.1103/PhysRevLett.95.076806

3. Zabet-Khosousi, A.; Dhirani, A.-A. Chem. Rev. 2008, 108, 4072-4124. doi:10.1021/cr0680134

4. Pauly, M.; Dayen, J.-F.; Golubev, D.; Beaufrand, J.-B.; Pichon, B. P.; Doudin, B.; Bégin-Colin, S. Small 2012, 8, 108-115. doi:10.1002/smll.201100931

5. Dayen, J.-F.; Devid, E.; Kamalakar, M. V.; Golubev, D.; Guédon, C. M.; Faramarzi, V.; Doudin, B.; Van der Molen, S. J. Adv. Mater. 2013, 25 , 400-404. doi:10.1002/adma.201201550

6. Andres, R. P.; Bielefeld, J. D.; Henderson, J. I.; Janes, D. B.; Kolagunta, V. R.; Kubiak, C. P.; Mahoney, W. J.; Osifchin, R. G. Science 1996, 273, 1690-1693. doi:10.1126/science.273.5282.1690

7. Lin, X. M.; Jaeger, H. M.; Sorensen, C. M.; Klabunde, K. J. J. Phys. Chem. B 2001, 105, 3353-3357. doi:10.1021/jp0102062

8. Liao, J.; Bernard, L.; Langer, M.; Schönenberger, C.; Calame, M. Adv. Mater. 2006, 18, 2444-2447. doi:10.1002/adma.200601001

9. Bernard, L.; Kamdzhilov, Y.; Calame, M.; Van der Molen, S. J.; Liao, J.; Schönenberger, C. J. Phys. Chem. C 2007, 111, 18445-18450. doi:10.1021/jp077095c

10. Hostetler, M. J.; Templeton, A. C.; Murray, R. W. Langmuir 1999, 15, 3782-3789. doi:10.1021/la981598f

11. Liao, J.; Agustsson, J. S.; Wu, S.; Schönenberger, C.; Calame, M.; Leroux, Y.; Mayor, M.; Jeannin, O.; Ran, Y.-F.; Liu, S.-X.; Decurtins, S. Nano Lett. 2010, 10, 759-764. doi:10.1021/n1902000e

12. Van der Molen, S. J.; Liao, J.; Kudernac, T.; Agustsson, J. S.; Bernard, L.; Calame, M.; van Wees, B. J.; Feringa, B. L.; Schönenberger, C. Nano Lett. 2009, 9, 76-80. doi:10.1021/nl802487j

13. Kaminska, A.; Inya-Agha, O.; Forster, R. J.; Keyes, T. E. Phys. Chem. Chem. Phys. 2008, 10, 4172-4180. doi:10.1039/b803007c

14. Halcrow, M. Coord. Chem. Rev. 2005, 249, 2880-2908. doi:10.1016/j.ccr.2005.03.010

15. Rajadurai, C.; Schramm, F.; Brink, S.; Fuhr, O.; Ghafari, M.; Kruk, R.; Ruben, M. Inorg. Chem. 2006, 45, 1019-1021. doi:10.1021/ic0612350

16. Cavallini, M.; Bergenti, I.; Militta, S.; Kengne, J. C.; Gentili, D.; Ruani, G.; Salitros, I.; Meded, V.; Ruben, M. Langmuir 2011, 27, 4076-4081. doi:10.1021/la104901m

17. Meded, V.; Bagrets, A.; Fink, K.; Chandrasekar, R.; Ruben, M.; Evers, F.; Bernand-Mantel, A.; Seldenthuis, J. S.; Beukman, A.; van der Zant, H. S. J. Phys. Rev. B 2011, 83, 245415. doi:10.1103/PhysRevB.83.245415

18. Chandrasekar, R.; Schramm, F.; Fuhr, O.; Ruben, M. Eur. J. Inorg. Chem. 2008, 17, 2649-2653. doi:10.1002/ejic.200800212

19. Kamenetska, M.; Quek, S. Y.; Whalley, A. C.; Steigerwald, M. L.; Choi, H. J.; Louie, S. G.; Nuckolls, C.; Hybertsen, M. S.; Neaton, J. B.; Venkataraman, L. J. Am. Chem. Soc. 2010, 132, 6817-6821. doi:10.1021/ja1015348

20. Guédon, C. M.; Zonneveld, J.; Valkenier, H.; Hummelen, J. C.; Van der Molen, S. J. Nanotechnology 2011, 22, 125205. doi:10.1088/0957-4484/22/12/125205
21. Nuzzo, R. G.; Allara, D. L. J. Am. Chem. Soc. 1983, 105, 4481-4483. doi:10.1021/ja00351a063

22. Tour, J. M.; Jones, L., II; Pearson, D. L.; Lamba, J. J. S.; Burgin, T. P.; Whitesides, G. M.; Allara, D. L.; Parikh, A. N.; Atre, S.

J. Am. Chem. Soc. 1995, 117, 9529-9534. doi:10.1021/ja00142a021

23. Huang, S.; Tsutsui, G.; Sakaue, H.; Shingubara, S.; Takahagi, T. J. Vac. Sci. Technol., B 2001, 19, 2045-2049. doi:10.1116/1.1410943

24. Santhanam, V.; Andres, R. P. Nano Lett. 2004, 4, 41-44. doi:10.1021/nl034851r

25. Dayen, J.-F.; Faramarzi, V.; Pauly, M.; Kemp, N. T.; Barbero, M.; Pichon, B. P.; Majjad, H.; Begin-Colin, S.; Doudin, B. Nanotechnology 2010, 21, 335303. doi:10.1088/0957-4484/21/33/335303

26. Midgley, P. A.; Weyland, M.; Thomas, J. M.; Johnson, B. F. G. Chem. Commun. 2001, 10, 907-908. doi:10.1039/b101819c

27. Kübel, C.; Voigt, A.; Schoenmakers, R.; Otten, M.; Su, D.; Lee, T.-C.; Carlsson, A.; Bradley, J. Microsc. Microanal. 2005, 11, 378-400. doi:10.1017/S1431927605050361

28. Kumar, J.; Wei, X.; Barrow, S.; Funston, A. M.; Thomas, K. G.; Mulvaney, P. Phys. Chem. Chem. Phys. 2013, 15, 4258-4264. doi:10.1039/c3cp44657c

29. Mie, G. Ann. Phys. 1908, 25, 377-445. doi:10.1002/andp.19083300302

30. Bernard, L. Expanding the Horizon of Molecular Electronics via Nanoparticle Assemblies. Ph.D. Thesis, University of Basel, Switzerland, 2006.

31. Garnett, J. M. Philos. Trans. R. Soc. London 1904, 203, 385-420. doi:10.1098/rsta.1904.0024

32. Cohen, R. W.; Cody, G. D.; Coutts, M. D.; Abeles, B. Phys. Rev. B 1973, 8, 3689-3701. doi:10.1103/PhysRevB.8.3689

33. Dalascu, D.; Martinu, L. J. Appl. Phys. 2000, 87, 228-235. doi:10.1364/JOSAB.18.000085

34. Camillone, N., III; Leung, T. Y. B.; Schwartz, P.; Eisenberger, P.; Scoles, G. Langmuir 1996, 12, 2737-2746. doi:10.1021/la951097j

35. Saikin, S. K.; Olivares-Amaya, R.; Rappoport, D.; Stopa, M.; Aspuru-Guzik, A. Phys. Chem. Chem. Phys. 2009, 11, 9401-9411. doi:10.1039/b906885f

36. Biggs, K. B.; Camden, J. P.; Anker, J. N.; Van Duyne, R. P. J. Phys. Chem. A 2009, 113, 4581-4586. doi:10.1021/jp8112649

37. Kang, X.; Chen, S. Nanoscale 2012, 4, 4183-4189. doi:10.1039/c2nr30213f

38. Cardini, G.; Muniz-Miranda, M. J. Phys. Chem. B 2002, 106, 6875-6880. doi:10.1021/jp014205I

39. Lordan, F.; Al-Attar, N.; Mallon, C.; Bras, J.; Collet, G.; Forster, R. J.; Keyes, T. E.; Rice, J. H. Chem. Phys. Lett. 2013, 556, 158-162. doi:10.1016/j.cplett.2012.11.028

40. Yan, H.; Lim, S. I.; Zhang, L.-C.; Gao, S.-C.; Mott, D.; Le, Y.; Loukrakpam, R.; An, D.-L.; Zhong, C.-J. J. Mater. Chem. 2011, 21 , 1890-1901. doi:10.1039/C0JM02772C

41. Eisler, S.; McDonald, R.; Loppnow, G. R.; Tykwinski, R. R. J. Am. Chem. Soc. 2000, 122, 6917-6928. doi:10.1021/ja000617g

42. Tran, T. B.; Beloborodov, I. S.; Hu, J.; Lin, X. M.; Rosenbaum, T. F.; Jaeger, H. M. Phys. Rev. B 2008, 78, 075437-075445. doi:10.1103/PhysRevB.78.075437

43. Shipway, A. N.; Katz, E.; Willner, I. ChemPhysChem 2000, 1, 18-52. doi:10.1002/1439-7641(20000804)1:1<18::AID-CPHC18>3.0.CO;2-L

44. Schmid, G.; Peschel, S.; Sawitowski, T. Z. Anorg. Allg. Chem. 1997, 623, 719-723. doi:10.1002/zaac.199762301114

45. Sato, T.; Brown, D.; Johnson, B. F. G. Chem. Commun. 1997, 1007-1008. doi:10.1039/A701627A 
46. George, C.; Szleifer, I.; Ratner, M. ACS Nano 2013, 7, 108-116. doi:10.1021/nn303320w

\section{License and Terms}

This is an Open Access article under the terms of the Creative Commons Attribution License

(http://creativecommons.org/licenses/by/2.0), which permits unrestricted use, distribution, and reproduction in any medium, provided the original work is properly cited.

The license is subject to the Beilstein Journal of Nanotechnology terms and conditions:

(http://www.beilstein-journals.org/bjnano)

The definitive version of this article is the electronic one which can be found at:

doi:10.3762/bjnano.5.177 